\title{
An Adaptive and Efficient Selective Multiple Reference Frames Motion Estimation for H.264 Video Coding**
}

\author{
Yu-Ming Lee, Yong-Fu Wang, Jia-Ren Wang, and Yinyi Lin \\ Department of Communication Engineering, \\ National Central University, Taiwan 32054 \\ \{yuming 0727, cyee01, kevinwang72326\} @gmail.com, \\ yilin@ce.ncu.edu.tw
}

\begin{abstract}
In the popular video coding standard H.264/AVC, many advanced techniques are employed. One important technique is the use of multiple reference frames motion estimation. However, the computational load increases with the number of references frames. In this paper, we suggest a selective multiple reference frames motion estimation (SMRFME) architecture which takes use of the information of the $1^{\text {st }}$ reference frame to determine whether it is necessary to search remaining reference frames. In addition, three early termination schemes are applied to the remaining reference frames of the candidate modes. The simulation results demonstrate that the proposed algorithm can achieve up to $77 \%$ of time saving compared to the multiple reference frames full search algorithm, while maintaining a high coding performance.
\end{abstract}

Keywords: H.264, multiple reference frames, selective multiple reference frames motion estimation (SMRFME), early termination, AZB, region based.

\section{Introduction}

The international video coding standard H.264/AVC has been approved by ITU-T as recommendation H.264 and by ISO/IEC as international standard MPEG-4 part 10 advanced video coding (AVC) [1]. The state-of-art H.264/AVC achieves significantly better performance in both PSNR and video quality at the same bit-rate compared with prior video coding standards. The improvement is typically $2-3 \mathrm{~dB}$ in PSNR, or equivalently $40 \%-60 \%$ in bit-rate reduction. One important technique is the use of multiple reference frames motion estimation (ME), and the computational complexity of H.264/AVC increases with the number of reference frames employed. The full selection procedure provides the best coding efficiency, but the five-fold increase in computation load is unbearable.

Many fast and efficient multi-frames ME algorithms have been investigated in recent years to reduce the computation cost and maintain coding performance. Some algorithms attempt to use context-based or histogram-similarity based method to

\footnotetext{
* This work was supported by the National Science Council, R.O.C. under Grant Number NSC 96-2221-E-008-013-MY2.
} 
speed up the multiple reference frames ME [2]. Some other algorithms attempt to reduce the computation using various prediction methods for selecting the initial search point [3]-[4]. In most of researches investigated perform the multiple reference frames ME mode by mode and select the best macroblock (MB) mode by considering the rate-distortion (RD) optimization technique. It is observed that most of the MB modes are finally predicted using the first reference frames, and just few of them are predicted with other reference frames. Another observation is that if the MB mode predicted using the $1^{\text {st }}$ frame has a bad motion cost (MCOST is shown in equation 1), it is then less likely to be the best mode predicted from its other reference frames. In this paper, based on these observations we propose a selective multiple reference frames ME (SMRFME) scheme which performs the $1^{\text {st }}$ reference frame ME first and performs remaining reference frames only for those modes with good MCOST predicted using the $1^{\text {st }}$ reference frame.

$$
\operatorname{MCOST}(s, c)=\operatorname{SAD}(s, c)+\lambda_{\text {MOTION }} \cdot \operatorname{Bit}(\Delta M V)
$$

where $S A D(s, c)$ is the sum of absolute differences between original block $s$ and candidate matching block $c . \lambda_{\text {MотіON }}$ is the Lagrange multiplier for ME. $\Delta M V$ is the difference between the predicted MV and the actual MV. Bit $(\Delta M V)$ is the number of bits representing the $\triangle M V$.

\section{Statistical Analysis of Multi-Reference Frames ME (MRFME)}

In the inter mode decision, 7 different block-size modes, varying among $16 \times 16\left(\mathrm{~m}_{1}\right)$, $16 \times 8\left(\mathrm{~m}_{2}\right), 8 \times 16\left(\mathrm{~m}_{3}\right), 8 \times 8\left(\mathrm{~m}_{4}\right), 8 \times 4\left(\mathrm{~m}_{5}\right), 4 \times 8\left(\mathrm{~m}_{6}\right)$ and $4 \times 4\left(\mathrm{~m}_{7}\right)$, are performed in each $\mathrm{MB}$ to achieve the best coding efficiency, in addition to the skip mode $\left(\mathrm{m}_{0}\right)$. These modes form a two-level hierarchy inside a MB. The first level L1 includes modes of $16 \times 16,16 \times 8,8 \times 16$, while the second level L2 includes modes of $8 \times 8,8 \times 4,4 \times 8$ and $4 \times 4$. In the H.264/AVC reference software baseline encoder, the multiple reference frames ME is conventionally performed mode by mode with full search of five reference frames, as illustrated in Fig. 1.

In most video sequences lots of background or motionless MBs are finally determined as a skip mode or L1 modes after computationally expensive rate distortion optimization. The MBs belonging to a high detail or fast motion area must be further split into L2 modes to get the best coding efficiency. In addition, most of MB modes end up with being predicted using the $1^{\text {st }}$ reference frame. An intensive experiment was conducted on many CIF and QCIF video sequences, to evaluate the distribution of best reference frame in each sequence.

The results conducted on 10 test video sequences are displayed in Table 1 for $\mathrm{QP}=28$. As shown, most of $\mathrm{MBs}$ are predicted from $1^{\text {st }}$ reference frame $(78 \%)$. We have observed that the MB mode, with a bad MCOST on the $1^{\text {st }}$ frame prediction, is then less likely to be the best MB mode predicted from its other reference frames. 


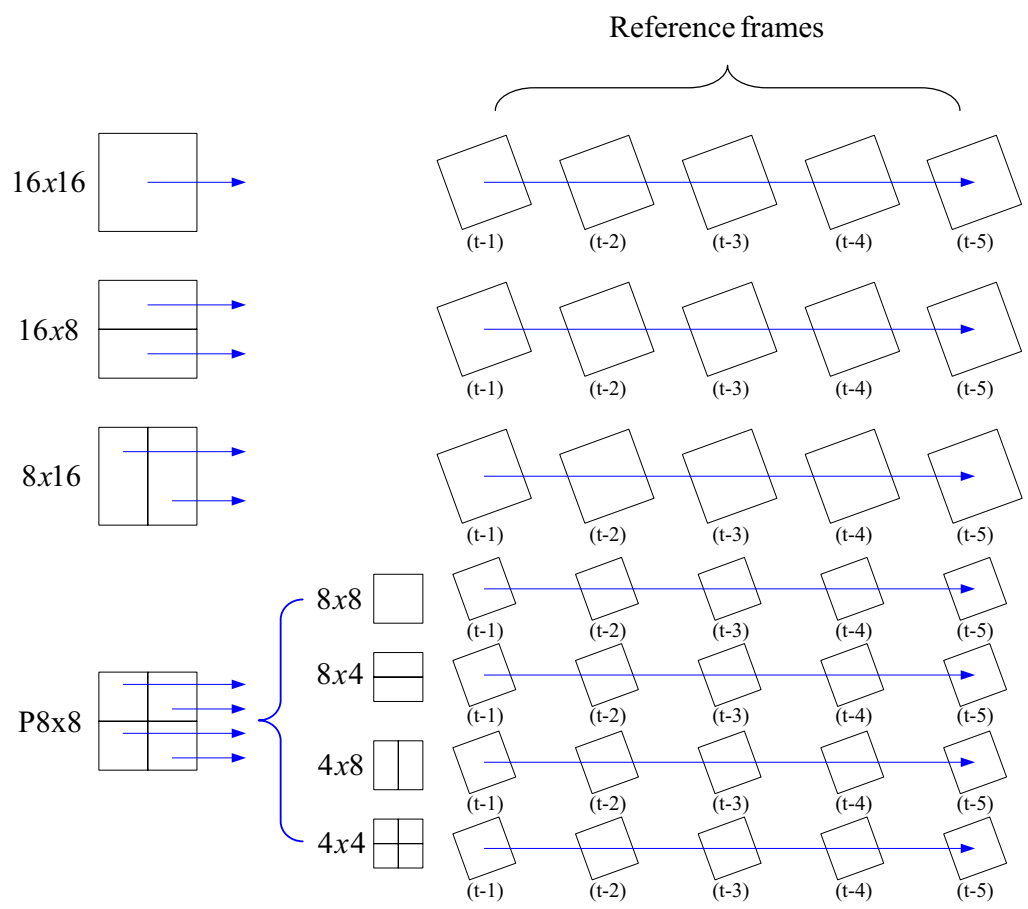

Fig. 1. Conventional Multi-Reference Frames Motion Estimation

Table 1. The distribution of the best reference frame

\begin{tabular}{|c|c|c|c|c|c|c|}
\hline \multicolumn{2}{|c|}{ QP=28 } & \multicolumn{5}{|c|}{ Distribution of the best reference frame } \\
\hline \multicolumn{2}{|c|}{ sequence } & $\mathbf{t}-\mathbf{1}$ & $\mathbf{t}-\mathbf{2}$ & $\mathbf{t}-\mathbf{3}$ & $\mathbf{t}-\mathbf{4}$ & $\mathbf{t}-\mathbf{5}$ \\
\hline \multirow{4}{*}{ QCIF } & claire & 96.70 & 1.18 & 1.10 & 0.35 & 0.68 \\
\cline { 2 - 7 } & grandma & 96.32 & 1.46 & 1.34 & 0.35 & 0.53 \\
\cline { 2 - 7 } & foreman & 73.50 & 11.68 & 8.72 & 3.32 & 2.77 \\
\cline { 2 - 7 } & football & 78.52 & 8.95 & 6.28 & 3.14 & 3.12 \\
\cline { 2 - 7 } & carphone & 75.66 & 8.75 & 8.41 & 3.54 & 3.65 \\
\hline \multirow{4}{*}{ CIF } & container & 91.68 & 3.69 & 2.07 & 1.40 & 1.17 \\
\cline { 2 - 7 } & tempete & 54.74 & 14.55 & 16.17 & 7.69 & 6.84 \\
\cline { 2 - 7 } & mobile & 46.87 & 16.14 & 16.86 & 10.32 & 9.81 \\
\cline { 2 - 7 } & paris & 93.13 & 3.51 & 2.01 & 0.78 & 0.57 \\
\cline { 2 - 7 } & stefan & 72.36 & 10.79 & 9.51 & 3.89 & 3.46 \\
\hline \multicolumn{2}{|c|}{ Avg. } & 77.95 & 8.07 & 7.25 & 3.48 & 3.26 \\
\hline
\end{tabular}

\section{Proposed Fast MRFME Algorithm}

Based on the analysis above, we propose a fast MRFME algorithm which takes use of the characteristics of the video sequences.

\subsection{Selective MRFME Scheme (SMRFME)}

From the statistical analysis described previously, the conventional multiple reference frames ME proposed in the reference software encoder, checking all reference frames 
equally likely for each mode, might not be an efficient scheme. To reduce the computation cost and maintain coding performance several fast and efficient multiple reference frames ME algorithms have been investigated in recent years. In [2], the context-based method was proposed to speed up the multiple reference frames ME. In [2], after the $1^{\text {st }}$ frame for all seven modes are performed, several context-based adaptive criteria are used to determine whether it is necessary to search the next reference frames. The procedure repeats until all 5 reference frames have been checked. The algorithm shows more efficient than the conventional one due to that most of best MB modes have been obtained in the $1^{\text {st }}$ reference frame prediction (as can be seen in Table 1), and the prediction in all other reference frames can be skipped with negligible degradation.

The algorithm proposed in [2], to whatever extent it occurs, doesn't consider that the probability is very low for a MB mode with a bad MCOST predicted in the $1^{\text {st }}$ frame, which becomes the final best MB mode predicted from its other reference frames. To improve the computation efficiency, in this section we propose a selective multiple reference frames ME algorithm. The SMRFME scheme is illustrated in Fig. 2. The SMRFME scheme is briefly summarized as follows:

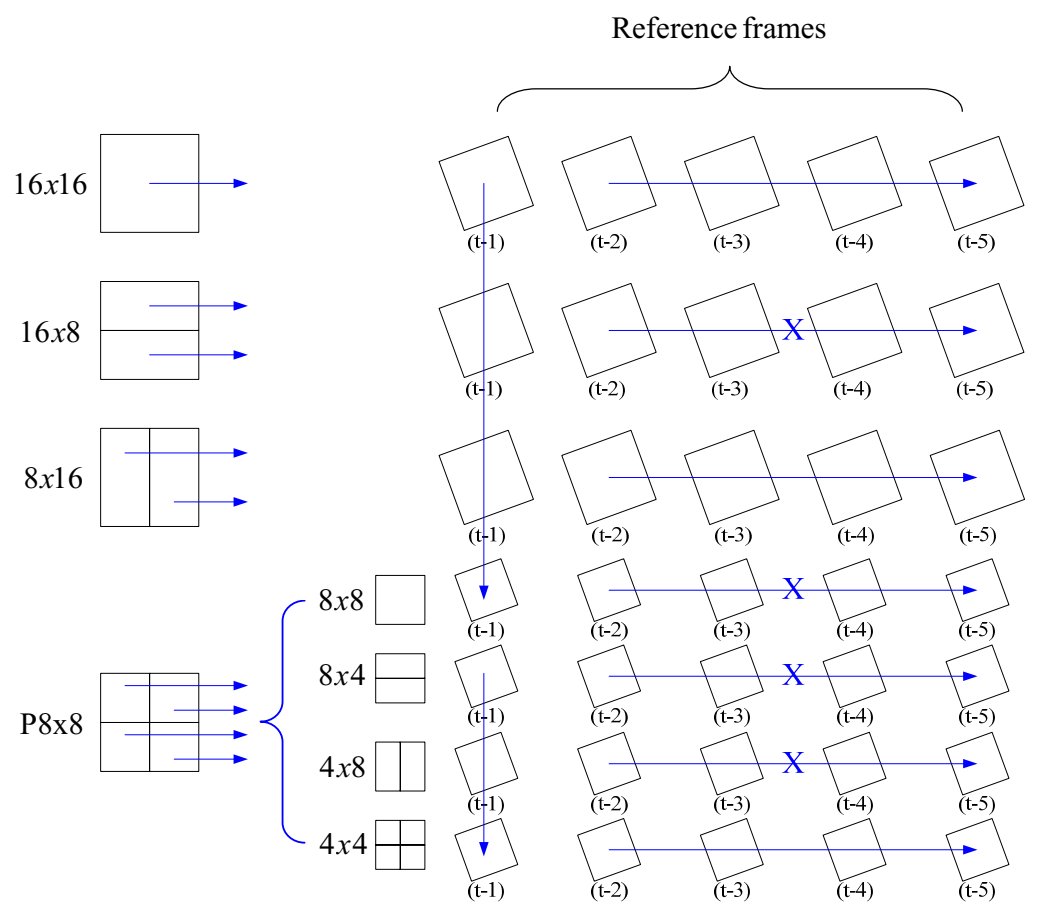

Fig. 2. Selective Multi-Reference Frames Motion Estimation

Assume $M C O S T_{1, b e s t}$ as the best MCOST predicted in the $1^{\text {st }}$ reference frame among all MB modes, which is given by

$$
\operatorname{MCOST}_{1, \text { est }}=\min \left\{\operatorname{MCOST}_{1, i}, i=0,1,2,3,4,5,6,7\right\}
$$


where $M C O S T_{1, i}$ represents the MCOST in mode $i$. Define the ratio of the MCOST, $\beta_{M \operatorname{COST}, i}$, as

$$
\beta_{M \operatorname{CoST}, i}=\frac{\operatorname{MCOST}_{1, i}}{M \operatorname{COST} T_{1, \text { best }}}
$$

To speed up the multiple reference frames decision process, after the $1^{\text {st }}$ reference frame prediction only the modes with $\beta_{M C O S T, i}$ less than a threshold $\beta$ are still performed ME for other reference frames. To determine the threshold $\beta$ for SMRFME, we examine the cumulative distribution function (CDF) of the best modes finally predicted from other reference frames that still can be acquired with this criterion, as a function of $\beta$ for several test sequences. The results are demonstrated in Fig. 3. As shown, a very large number of the best modes can be identified when $\beta=1.2$, i.e., the mode with $M C O S T$ value $M C O S T_{1, i} \geq 1.2 M C O S T_{1, b e s t}$ are unlikely to obtain the best reference frame in its remaining reference frames.

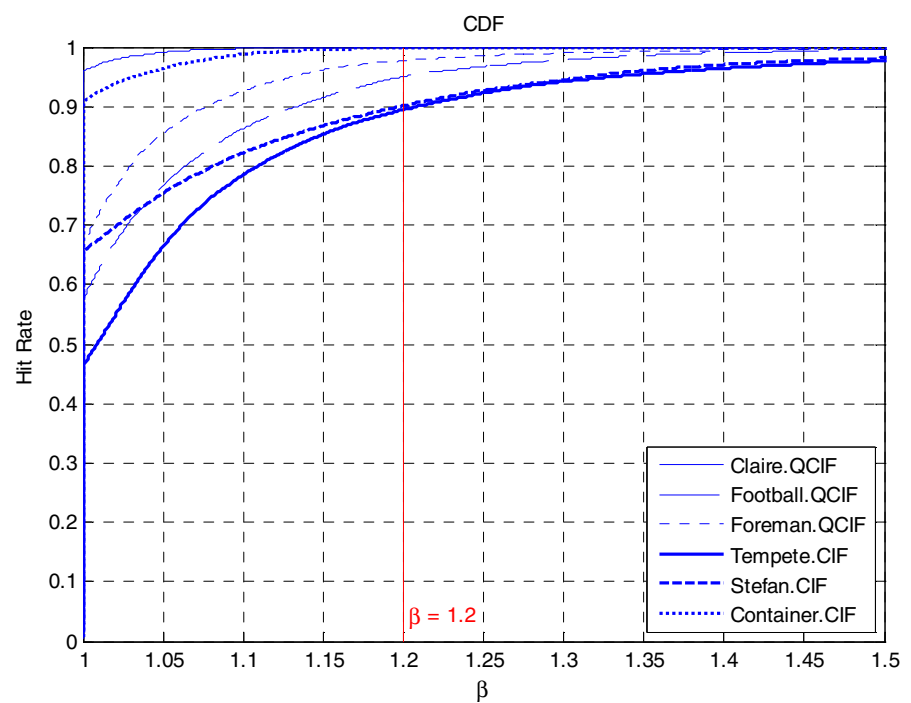

Fig. 3. CDF of the hit rate as a function of factor $(\beta)$

\subsection{Early Termination Algorithms for Remaining Reference Frames}

Though the SMRFME scheme can bring out good coding performance, it achieves $41 \%$ of computation reduction on average. Although the remaining reference frames of least possible modes are discarded, five reference frames of the candidate modes are still performed. In this section, three early termination algorithms are proposed to further quicken the process of searching reference frames. 


\subsubsection{Region Based Algorithm (Denoted as Region Based)}

Generally, the correlation between the adjacent frames is very strong. Our investigation revealed that the continuity of the motion can be explored in order to simplify the multireference frames selection. Assume an object is moving in a video sequence and keeps the similar appearance in adjacent frames. The best reference frame of previous frame can be used as the maximum reference frame of current frame. An example is given in Fig. 4, which illustrates the best reference frame for each macroblock in two successive encoded frames of Foreman, where 0 to 4 are the best reference index (ref_idx). It is clearly shown that the $\mathrm{MB}$ in frame $(\mathrm{t})$ are highly correlated with their co-located or surrounding MB in the frame $(t-1)$. Fig. 5 shows the MB in current and its co-located and neighbouring $\mathrm{MBs}$ in reference frame, where co-located (E') and surrounding (A-I) MBs form a region. For the frame $(\mathrm{t})$, we first check the ref_idx of the region in previously encoded frame $(\mathrm{t}-1)$. To avoid additional computation complexity, we just find out the maximum ref_idx in the region. We use the maximum ref_idx to determine the maximum searched frame for the frame $(\mathrm{t})$. The maximum searched frame is defined as

Maximum searched frame $=\max \left\{r e f \_i d x_{A}, r e f_{-} i d x_{B}, \ldots, r e f \_i d x_{I}\right\}$

\begin{tabular}{|l|l|l|l|l|l|l|l|l|l|l|}
\hline 0 & 0 & 0 & 2 & 0 & 0 & 0 & 1 & 0 & 0 & 0 \\
\hline 0 & 3 & 0 & 1 & 0 & 3 & 0 & 1 & 2 & 0 & 2 \\
\hline 0 & 3 & 3 & 0 & 0 & 0 & 0 & 0 & 2 & 2 & 0 \\
\hline 1 & 0 & 2 & 0 & 0 & 0 & 0 & 1 & 2 & 2 & 4 \\
\hline 0 & 0 & 2 & 0 & 0 & 0 & 0 & 0 & 2 & 2 & 2 \\
\hline 1 & 0 & 0 & 0 & 0 & 0 & 0 & 0 & 2 & 3 & 0 \\
\hline 0 & 0 & 0 & 0 & 0 & 2 & 2 & 0 & 2 & 2 & 2 \\
\hline 0 & 0 & 1 & 0 & 0 & 4 & 0 & 0 & 0 & 0 & 0 \\
\hline 0 & 0 & 0 & 0 & 0 & 0 & 0 & 0 & 0 & 0 & 0 \\
\hline \multicolumn{7}{|c|}{ Best ref_idx } \\
\hline
\end{tabular}

\begin{tabular}{|l|l|l|l|l|l|l|l|l|l|l|}
\hline 0 & 0 & 0 & 0 & 0 & 0 & 0 & 0 & 0 & 0 & 0 \\
\hline 0 & 0 & 3 & 2 & 2 & 0 & 0 & 3 & 3 & 4 & 0 \\
\hline 0 & 0 & 0 & 0 & 0 & 0 & 0 & 0 & 1 & 0 & 0 \\
\hline 0 & 0 & 2 & 0 & 0 & 0 & 0 & 0 & 0 & 1 & 0 \\
\hline 0 & 1 & 0 & 0 & 0 & 0 & 1 & 0 & 0 & 1 & 1 \\
\hline 0 & 1 & 0 & 0 & 0 & 0 & 0 & 0 & 0 & 1 & 1 \\
\hline 0 & 0 & 0 & 0 & 0 & 0 & 0 & 0 & 0 & 0 & 4 \\
\hline 0 & 0 & 0 & 0 & 0 & 0 & 0 & 0 & 0 & 0 & 0 \\
\hline 0 & 0 & 0 & 0 & 0 & 0 & 0 & 0 & 0 & 1 & 1 \\
\hline \multicolumn{10}{|c|}{ Best ref_idx in frame } & $(\mathrm{t})$ \\
\hline
\end{tabular}

Fig. 4. The ref_idx of the two adjacent frames in Foreman

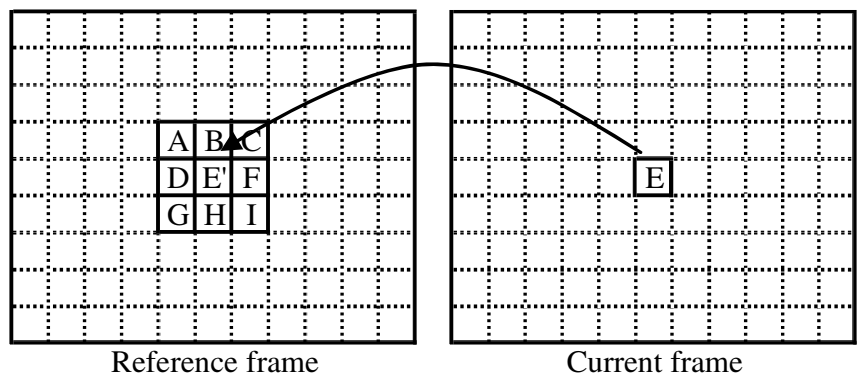

Fig. 5. Region based macroblocks

\subsubsection{Detection of All Zero Coefficients Algorithm (Denoted as AZB)}

In motion estimation process, the SAD between the current block and its best matching block can be used to detect all zero blocks. If it is detected that the transformed and quantized coefficients are very close to zero, the remaining reference frames can 
be terminated. In order to save the DCT and quantization, a nearly sufficient condition for early zero-block detection is derived [5]. If all the SAD blocks are smaller than threshold $\left(3.5 \cdot Q_{\text {step }}\right)$, the ME process of the remaining reference frames is terminated immediately.

\subsubsection{Detection of Monotonic Increment Algorithm (Denoted as Monotonic)}

We can see form Table 1 that the probability that $\operatorname{ref}_{\mathrm{t}-1}, \operatorname{ref}_{\mathrm{t}-2}$ and $\mathrm{ref}_{\mathrm{t}-3}$ are selected as the best reference frame is very high, more than $93 \%$ on average. Thus, the MCOSTs of $\mathrm{ref}_{\mathrm{t}-1}, \mathrm{ref}_{\mathrm{t}-2}$ and $\mathrm{ref}_{\mathrm{t}-3}$ can also be used to determine whether or not search $\mathrm{ref}_{\mathrm{t}-4}$ and ref $_{\mathrm{t}-5}$. When the MCOST of $\mathrm{ref}_{\mathrm{t}-3}$ is largest among the first three reference frames, these three MCOSTs will most likely be monotonic increment. The probability that $\mathrm{ref}_{\mathrm{t}-4}$ and $\mathrm{ref}_{\mathrm{t}-5}$ selected as the best reference frame is very little. For this condition, the ME procedure of the $\mathrm{ref}_{\mathrm{t}-4}$ and $\mathrm{ref}_{\mathrm{t}-5}$ is omitted.

\subsubsection{Analysis of Our Proposed Early Termination Algorithms}

The hit rate analysis of these three algorithms is shown in Table 3. It can be seen that the average hit rate of these three algorithms reaches $95 \%, 89 \%$ and $97 \%$, respectively. Thus, we can get the good coding performance in simulation.

Table 3. The hit rate analysis

\begin{tabular}{|c|c|c|c|c|}
\hline \multicolumn{2}{|c|}{ Sequence } & Region based & AZB & Monotonic \\
\hline \multirow{6}{*}{ QCIF } & football & $94.51 \%$ & $92.89 \%$ & $95.24 \%$ \\
\hline & coastguard & $94.01 \%$ & $91.98 \%$ & $98.37 \%$ \\
\hline & trevor & $95.69 \%$ & $93.19 \%$ & $99.28 \%$ \\
\hline & claire & $97.58 \%$ & $98.03 \%$ & $99.42 \%$ \\
\hline & foreman & $92.49 \%$ & $83.60 \%$ & $96.58 \%$ \\
\hline & tennis & $96.04 \%$ & $90.09 \%$ & $97.73 \%$ \\
\hline \multirow{6}{*}{ CIF } & bus & $94.42 \%$ & $88.56 \%$ & $97.30 \%$ \\
\hline & dancer & $94.31 \%$ & $91.32 \%$ & $98.69 \%$ \\
\hline & waterfall & $93.00 \%$ & $89.59 \%$ & $97.14 \%$ \\
\hline & news & $97.16 \%$ & $96.52 \%$ & $99.39 \%$ \\
\hline & stefan & $93.27 \%$ & $84.88 \%$ & $96.18 \%$ \\
\hline & mobile & $97.18 \%$ & $72.23 \%$ & $89.01 \%$ \\
\hline \multicolumn{2}{|c|}{ AVG. } & $94.97 \%$ & $89.41 \%$ & $97.03 \%$ \\
\hline
\end{tabular}

\section{Experimental Results}

To evaluate the performance of the efficient selective multiple reference frames $\mathrm{ME}$ algorithm, our proposed algorithms were implemented in H.264 reference encoder JM12.2. The proposed algorithms were tested on 4 QCIF (176x144) and CIF $(352 \times 288)$ sequences which represent different motion activities. The test conditions for simulation are given as follows:

Table 4. Simulation condition

\begin{tabular}{|c|c|c|c|}
\hline \# of coded frames & 100 & GOP structure & IPPP... \\
\hline \# of reference frames & 5 & Search range & \pm 16 \\
\hline RDO & on & QP & $20,24,28,32,36$ \\
\hline Entropy coding & CAVLC & Resolution & $1 / 4$ pixel \\
\hline Intra mode & on & Inter mode & on \\
\hline
\end{tabular}


Table 5. Performance comparison for $\mathrm{QP}=28$

(a) PSNR comparison

\begin{tabular}{|c|c|c|c|c|c|c|c|}
\hline \multicolumn{2}{|c|}{ QP=28 } & \multicolumn{7}{c|}{ PSNR comparison $(\mathbf{d B}, \triangle(\mathbf{d B}))$} \\
\hline \multicolumn{2}{|c|}{ Sequence } & Orig. & SMRFME & Rgeion based & AZB & Monotonic & Proposed \\
\hline \multirow{4}{*}{ QCIF } & football & 34.547 & -0.011 & -0.028 & -0.009 & -0.018 & -0.023 \\
\cline { 2 - 8 } & coastguard & 34.682 & -0.013 & -0.025 & 0.006 & -0.017 & -0.035 \\
\cline { 2 - 8 } & trevor & 36.912 & 0.003 & -0.008 & -0.023 & 0.000 & -0.016 \\
\cline { 2 - 8 } & claire & 39.823 & 0.061 & 0.004 & 0.008 & 0.007 & -0.015 \\
\hline \multirow{4}{*}{ CIF } & bus & 35.718 & -0.017 & -0.020 & -0.005 & -0.006 & -0.037 \\
\cline { 2 - 8 } & dancer & 40.609 & -0.001 & -0.003 & -0.017 & -0.001 & -0.025 \\
\cline { 2 - 8 } & waterfall & 35.454 & -0.004 & -0.036 & 0.004 & -0.029 & -0.052 \\
\cline { 2 - 8 } & news & 38.585 & -0.013 & -0.020 & -0.016 & -0.016 & -0.033 \\
\hline \multicolumn{6}{|c|}{} & \multicolumn{7}{|c|}{0.001} & -0.017 & -0.006 & -0.010 & -0.030 \\
\hline
\end{tabular}

(b) Bit-rate comparison

\begin{tabular}{|c|c|c|c|c|c|c|c|}
\hline \multicolumn{2}{|c|}{ QP=28 } & \multicolumn{7}{c|}{ Bit rate comparison (bps, $\triangle(\%)$ ) } \\
\hline \multicolumn{2}{|c|}{ Sequence } & Orig. & SMRFME & Rgeion based & AZB & Monotonic & Proposed \\
\hline \multirow{4}{*}{ QCIF } & football & 1060999 & 0.41 & 0.08 & -0.03 & 0.03 & 0.59 \\
\cline { 2 - 8 } & coastguard & 279156 & 0.37 & 0.55 & 0.46 & 0.00 & 0.58 \\
\cline { 2 - 8 } & trevor & 142279 & 0.12 & 0.02 & -0.18 & -0.37 & 0.07 \\
\cline { 2 - 8 } & claire & 32599 & -0.39 & 1.71 & -0.46 & -0.04 & 0.10 \\
\hline \multirow{4}{*}{ CIF } & bus & 1328657 & 1.05 & 0.08 & -0.08 & 0.04 & 1.50 \\
\cline { 2 - 8 } & dancer & 608738 & 0.11 & -0.15 & 0.10 & -0.05 & 0.39 \\
\cline { 2 - 8 } & waterfall & 324540 & -0.08 & 0.51 & 0.18 & 0.48 & 0.64 \\
\cline { 2 - 8 } & news & 227602 & -0.01 & 0.01 & 0.00 & 0.10 & 0.40 \\
\hline \multicolumn{2}{|c|}{ Avg. } & \multicolumn{7}{|c|}{0.20} & 0.35 & 0.00 & 0.02 & 0.53 \\
\hline
\end{tabular}

(c) Time comparison

\begin{tabular}{|c|c|c|c|c|c|c|c|}
\hline \multicolumn{2}{|c|}{ QP=28 } & \multicolumn{7}{c|}{ Time comparison $(\mathbf{m s ,} \triangle(\boldsymbol{\%}))$} \\
\hline \multicolumn{2}{|c|}{ Sequence } & Orig. & SMRFME & Rgeion based & AZB & Monotonic & Proposed \\
\hline \multirow{4}{*}{ QCIF } & football & 137340 & -43.37 & -15.89 & -5.11 & -25.60 & -60.36 \\
\cline { 2 - 8 } & coastguard & 108387 & -41.77 & -44.09 & -10.60 & -32.52 & -65.41 \\
\cline { 2 - 8 } & trevor & 76118 & -39.52 & -42.04 & -20.13 & -31.95 & -67.74 \\
\cline { 2 - 8 } & claire & 49666 & -48.18 & -47.68 & -29.38 & -31.71 & -71.20 \\
\hline \multirow{4}{*}{ CIF } & bus & 468038 & -44.60 & -26.50 & -13.13 & -27.86 & -64.85 \\
\cline { 2 - 8 } & dancer & 380253 & -41.18 & -46.70 & -38.39 & -36.97 & -77.81 \\
\cline { 2 - 8 } & waterfall & 347551 & -38.85 & -22.42 & -15.15 & -21.72 & -56.07 \\
\cline { 2 - 8 } & news & 237827 & -35.68 & -46.11 & -27.84 & -31.42 & -68.91 \\
\hline \multicolumn{2}{|c|}{ Avg. } & & -41.64 & -36.43 & -19.97 & -29.97 & -66.54 \\
\hline
\end{tabular}

The simulation results of PSNR gain, bit-rate increment and coding time compared to multiple reference frames full search algorithm (denoted as Orig.) are shown in Table 5. The algorithm which combines SMRFME algorithm with all early termination algorithms is denoted as proposed algorithm. The simulation results show that our proposed algorithm achieves $66 \%$ coding time on average. Meanwhile the performance of our proposed algorithm is almost the same as the Orig., with negligible loss in PSNR ( $0.03 \mathrm{~dB}$ loss) and bit-rate ( $0.53 \%$ bit-rate increment).

Fig. 6 depicts the rate-distortion (RD) performance of sequence football and Stefan. Compared with Orig. and our proposed algorithm, the average PSNR drop of our algorithm is negligible. Therefore, the rate-distortion curve of our proposed algorithm and the Orig. algorithm are hardly distinguishable. Table 6 shows that the complexity reduction of our proposed algorithm in various bit-rate. Results also show that our proposed algorithm can reduce computation load efficiently, while keeping good coding performance. 
$\rightarrow$ Orig. $\rightarrow$ SMRFME $\rightarrow$ Region $\star$ AZB $\rightarrow$ Monotonic $\rightarrow$ Proposed

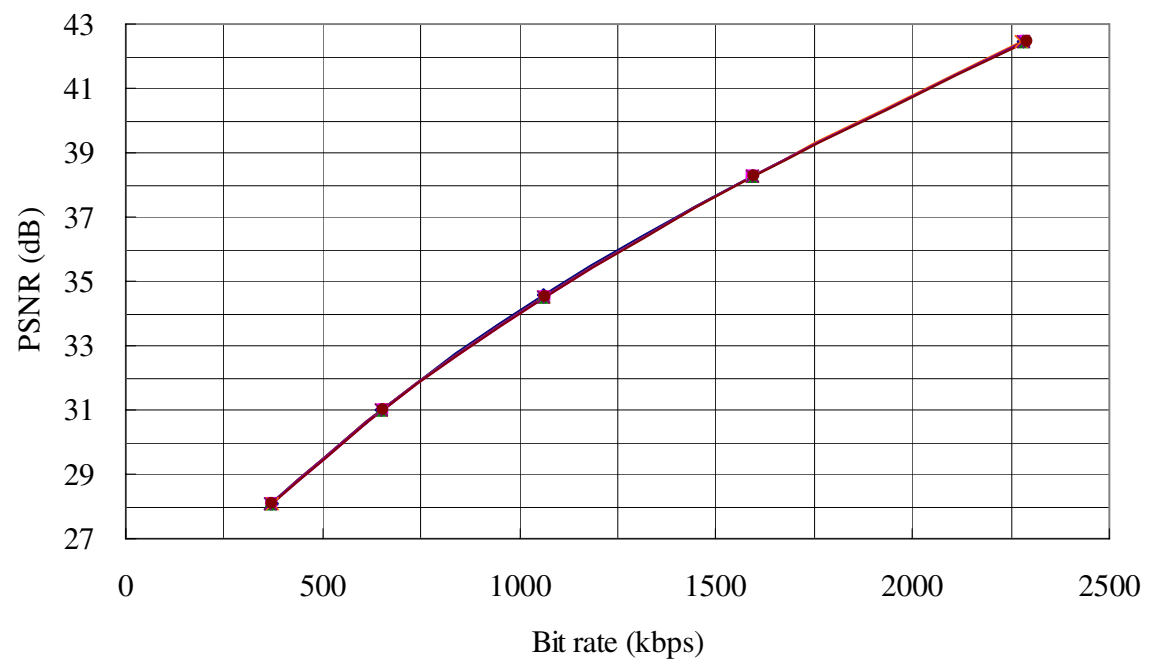

(a) Football.QCIF

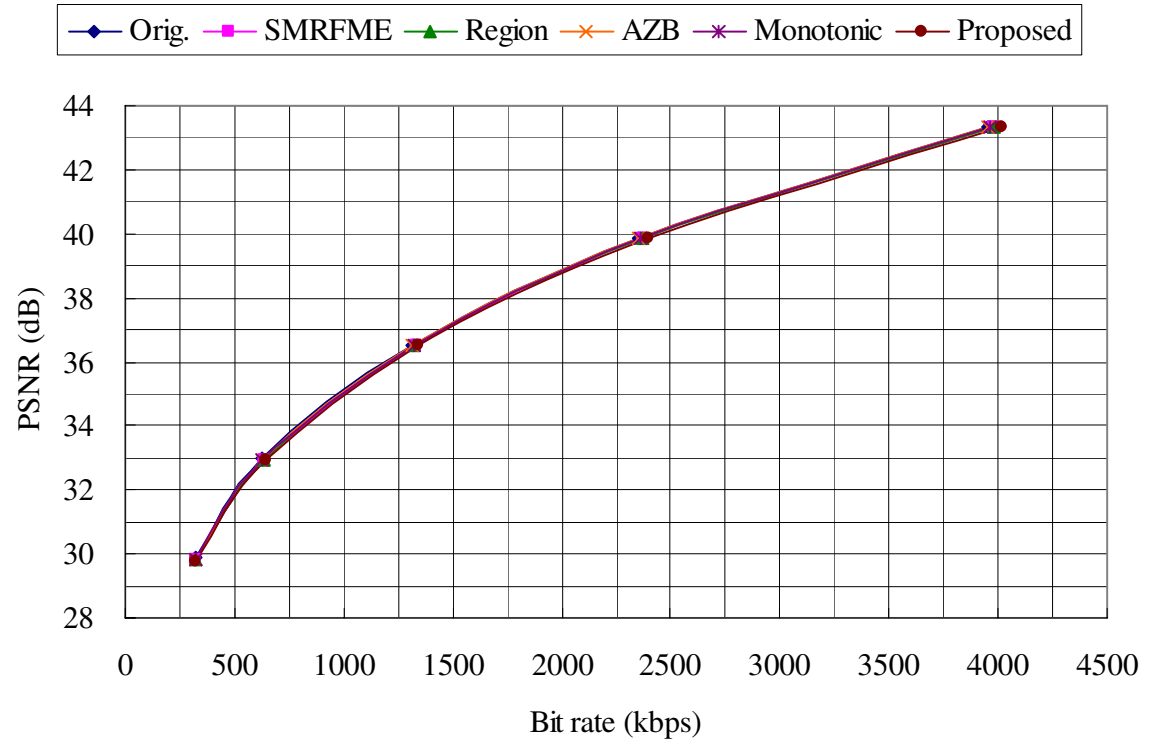

(b) Stefan.CIF

Fig. 6. Rate-distortion performance comparison 
Table 6. Coding efficiency comparison

(a) Football.QCIF

\begin{tabular}{|c|c|c|c|c|c|c|}
\hline Football.QCIF & \multicolumn{7}{|c|}{ Time comparison $(\mathbf{m s}, \triangle(\boldsymbol{\%}))$} \\
\hline $\mathbf{Q P}$ & Orig. & SMRFME & Rgeion based & AZB & Monotonic & Proposed \\
\hline $\mathbf{2 0}$ & 146613 & -44.17 & -15.25 & -0.27 & -24.73 & -60.89 \\
\hline $\mathbf{2 4}$ & 142596 & -44.30 & -14.87 & -1.60 & -25.31 & -61.04 \\
\hline $\mathbf{2 8}$ & 136813 & -43.34 & -15.94 & -4.96 & -25.42 & -60.74 \\
\hline $\mathbf{3 2}$ & 129417 & -40.96 & -18.16 & -11.51 & -25.88 & -60.32 \\
\hline $\mathbf{3 6}$ & 118116 & -37.66 & -24.48 & -16.71 & -26.86 & -62.59 \\
\hline
\end{tabular}

(b) Stefan.CIF

\begin{tabular}{|c|c|c|c|c|c|c|}
\hline Stefan.CIF & \multicolumn{7}{|c|}{ Time comparison $(\mathbf{m s}, \triangle(\%)$ ) } \\
\hline QP & Orig. & SMRFME & Rgeion based & AZB & Monotonic & Proposed \\
\hline $\mathbf{2 0}$ & 443593 & -41.67 & -17.66 & -8.93 & -22.51 & -60.72 \\
\hline $\mathbf{2 4}$ & 416228 & -41.67 & -22.16 & -12.09 & -23.30 & -62.92 \\
\hline $\mathbf{2 8}$ & 389335 & -42.63 & -25.80 & -13.74 & -24.40 & -64.41 \\
\hline $\mathbf{3 2}$ & 363736 & -44.18 & -31.59 & -16.63 & -26.50 & -66.16 \\
\hline $\mathbf{3 6}$ & 339981 & -43.76 & -41.35 & -20.99 & -29.31 & -68.86 \\
\hline
\end{tabular}

\section{Conclusion}

In this paper we present a fast algorithm for multiple reference frames motion estimation in H.264/AVC to reduce the computation. To skip unnecessary reference frames motion estimation, the proposed algorithm uses information of the previous search reference frames and the correlation among the neighboring blocks. Experimental results show that the proposed algorithm reduces the coding complexity notably while achieves similar gain as the multiple reference frames full search algorithm.

\section{References}

1. Wiegand, T., Sullivan, G.J., Bjontegaard, G., Luthra, A.: Overview of the H.264/AVC video coding standard. IEEE Trans. Circuits Syst. Video Technol. 13(7), 560-576 (2003)

2. Huang, Y.W., Hsieh, B.Y., Chien, S.Y., Ma, S.Y., Chen, L.G.: Analysis and Complexity Reduction of Multiple Reference Frames Motion Estimation in H.264/AVC. IEEE Trans. Circuits Syst. Video Technol. 16(7), 507-522 (2006)

3. Su, Y., Sun, M.T.: Fast multiple reference frame motion estimation for H.264/AVC. IEEE Trans. Circuits Syst. Video Technol. 16, 447-452 (2006)

4. Chen, M.J., Chiang, Y.Y., Li, H.J., Chi, M.C.: Efficient multi-frame motion estimation algorithm for MPEG-4 AVC/JVT/H.264. In: Proc. IEEE ISCAS, pp. III-737-III-740 (May 2004)

5. Lee, Y.M., Lin, Y.: An improved zero-block mode decision algorithm for H.264/AVC. In: Proc. IEEE ICIP, vol. 5, pp. V-293-V-296 (September 2007) 